\title{
Trends in Mathematics: How They Could Change Education
}

\author{
by László Lovász
}

\author{
Department of Computer Science, Eötvös Loránd University, Budapest, Hungary
}

Mathematical activity has changed a lot in the last 50 years. Some of these changes, like the use of computers, are very visible and are being implemented in mathematical education quite extensively. There are other, more subtle trends that may not be so obvious. We discuss some of these trends and how they could, or should, influence the future of mathematical education.

\section{Introduction}

Mathematical activity (research, applications, education, exposition) has changed a lot in the last 50 years. Some of these changes, like the use of computers, are very visible and are being implemented in mathematical education quite extensively. There are other, more subtle trends that may not be so obvious. Should these influence the way we teach mathematics? The answer may, of course, be different at the primary, secondary, undergraduate and graduate level.

Here are some of the general trends in mathematics, which we should take into account.

1. The size of the community and of mathematical research activity is increasing exponentially; it doubles every 25 years or so. This fact has a number of consequences: the impossibility of keeping up with new results; the need of more efficient cooperation between researchers; the difficulty of identifying "core" mathematics (to be mastered at various levels); the need for better dissemination of new ideas. How can mathematical education prepare future researchers and appliers of mathematics, future decision makers and the informed public for these changes?

2. New areas of application, and their increasing significance. Information technology, sciences, the economy, and almost all areas of human activity make more and more use of mathematics, and, perhaps more significantly, they use all branches of mathematics, not just traditional applied mathematics. How can we train our students to recognize problems where mathematics can help in the solution?

3. New tools: computers and information technology. This is perhaps the most visible new feature, and accordingly a lot has been done to introduce com- puters in education. But the influence of computers on our everyday life and research is also changing fast: besides the design of algorithms, experimentation, and possibilities in illustration and visualization, we use email, discussion groups, on-line encyclopedias and other internet resources. Can education utilize these possibilities, keep up with the changes, and also teach students to use them in productive ways?

4. New forms of mathematical activity. In part as an answer to the issues raised above, many new forms of mathematical activity are gaining significance: algorithms and programming, modeling, conjecturing, expository writing and lecturing. Which of these non-traditional mathematical activities could and should be taught to students?

I will say some more about these trends, and discuss the question of their influence on mathematical education. I will make use of some observations from my earlier articles $[6,7]$.

\section{The size of the community and of mathematical research activity}

The number of mathematical publications (along with publications in other sciences) has increased exponentially in the last 50 years. Mathematics has outgrown the small and close-knit community of nerds that it used to be; with increasing size, the profession is becoming more diverse, more structured and more complex.

Mathematicians sometimes pretend that mathematical research is as it used to be: that we find all the information that might be relevant by browsing through the new periodicals in the library, and that if we publish a paper in an established journal, then it will reach all the people whose research might utilize our results. But of course $3 / 4$ of the relevant periodicals are not on the library table, and even if one had access to all these journals, and had the time to read all of them, one would only be familiar with the results of a small corner of mathematics.

A larger structure is never just a scaled-up version of the smaller. In larger and more complex animals an increasingly large fraction of the body is devoted to "over- 
head": the transportation of material and the coordination of the function of various parts. In larger and more complex societies an increasingly large fraction of the resources is devoted to non-productive activities like transportation information processing, education or recreation.

We have to realize and accept that a larger and larger part of our mathematical activity will be devoted to communication.

This is easy to observe: the number of professional visits, conferences, workshops, research institutes is increasing fast, e-mail is used more and more. The percentage of papers with multiple authors has jumped. But probably we will reach the point soon where mutual personal contact does not provide sufficient information flow.

There is another consequence of the increase in mass: the inevitable formation of smaller communities, one might say subcultures. One response to this problem is the creation of an activity that deals with the secondary processing of research results. For lack of a better word, I'll call this expository writing, although I'd like to consider it more as a form of mathematical research than as a form of writing: finding the ramifications of a result, its connections with results in other fields, explaining, perhaps translating it for people coming from a different subculture.

Are there corresponding changes in mathematical curricula and, more generally, in the way we teach mathematics? The first, and most pressing, problem is the sheer size of material that would be nice (or absolutely necessary) to teach. In addition, as we will see, we should put more emphasis on (which also means giving more teaching time to) some non-traditional mathematical activities like algorithm design, modeling, experimentation and exposition. I also have to emphasize the necessity of preserving problem solving as a major feature of teaching mathematics.

How to find time to learning concepts, theorems, proofs, especially with the rapid expansion of material, and at a time when class time devoted to mathematics is being reduced in many countries? Which of the new areas should make its way to education (on the secondary or college level), and which of the traditional material should be left out? This is not a one-time crisis: mathematical research is not showing any signs of slowing down.

One possible answer to this question is to leave the teaching of any recently developed area of mathematics to later in the education, to Masters and $\mathrm{PhD}$ programs. The trouble with this approach is that many educated people will never meet the mathematics of the last 200 years, which will contribute to the unfortunate but persistent misconception that mathematics is a closed subject. Many of the new areas of mathematics are important for un- derstanding developments in technology and science, and by not teaching them we give up illuminating the increasing role of mathematics in modern life.

The other possible answer is to remove from the curriculum traditional material that is deemed less important. This approach has the negative effect of eroding well-established methods for teaching mathematical thinking. For example, elementary geometry has been purged from the curriculum in many countries. While this kind of geometry is indeed peripheral in modern mathematical research, it is of course still important in applications, and, perhaps even more important, its study is very instrumental in the development of spatial conception, and, perhaps even more significantly, in understanding the real nature of mathematical proofs, the "Aha" event when an incomprehensible connection becomes clear through looking at it the right way.

I have no easy answer to this question. Probably one must concentrate on mathematical competencies like problem solving, abstraction, generalization and specialization, logical reasoning and use of mathematical formalism, along with the non-traditional skills mentioned above (see e.g. [10]). One could select a mixture of classical and more modern mathematical topics that are best suited to develop these competencies and (of course) basic skills, and at the same time give some sort of picture of the historical roots as well as contemporary applications.

Another question raised by the increasing complexity of the world of mathematics is whether exposition style mathematics has any place in education. One aspect of this is teaching students to explain mathematics to "outsiders", teaching them how to summarize results without getting lost in the details. This is not easy to do, but to teach such skills would be very useful indeed.

A more heretical thought is to do some expository style teaching. In most sciences like chemistry or astronomy, it is natural to teach in high school or even college the facts without explaining all the technical details of their discovery (or even of their exact meaning). Some of this is done in mathematics too: many students learn that the regular pentagon can be constructed with ruler and compass but the regular heptagon cannot, or that equations of degree 5 or more cannot in general be solved by radicals. But these examples are almost 200 years old! Can we solve the problem of exposing students to modern mathematics by working out appropriate non-exact but still mathematical blocks of material? I hesitate to answer "YES", but the question is valid.

\section{New areas of application, and their increasing significance}

The traditional areas of application of mathematics are physics and engineering. The branch of mathematics 
used in these applications is analysis, primarily differential equations.

But in the boom of scientific research in the last 50 years, many other sciences have come to the point where they need serious mathematical tools, and quite often the traditional tools of analysis are not adequate.

For example, biology studies the genetic code, which is discrete: simple basic questions like finding matching patterns, or tracing consequences of flipping over substrings, sound more familiar to the combinatorialist than to the researcher of differential equations. A question about the information content, redundancy, or stability of the code may sound too vague to a classical mathematician but a theoretical computer scientist will immediately see at least some tools to formalize it (even if to find the answer may be too difficult at the moment).

Even physics has its encounters with unusual discrete mathematical structures: elementary particles, quarks and the like are very combinatorial; understanding basic models in statistical mechanics requires graph theory and probability.

Economics is a heavy user of mathematics-and much of its need is not part of the traditional applied mathematics toolbox. The success of linear programming in economics and operations research depends on conditions of convexity and unlimited divisibility; taking indivisibilities into account (for example, logical decisions, or individuals) leads to integer programming and other combinatorial optimization models, which are much more difficult to handle.

Finally, there is a completely new area of applied mathematics: computer science. The development of electronic computation provides a vast array of well-formulated, difficult, and important mathematical problems, raised by the study of algorithms, data bases, formal languages, cryptography and computer security, VLSI layout, and much more. Most of these have to do with discrete mathematics, formal logic, and probability.

One must add that which branches of mathematics will be applicable in the near future is utterly unpredictable. Just 30 years ago questions in number theory seemed to belong to the purest, most classical and completely inapplicable mathematics; now many areas in number theory belong to the core of mathematical cryptography and computer security.

A very positive development in recent decades is the decreasing separation between pure and applied mathematics. I feel that the mutual respect of pure and applied mathematicians is increasing, along with the number of people contributing to both sides. The diversity of applications should also strengthen the flow of information across all of mathematics.

No field can retreat into its ivory tower and close its doors to applications; nor can any field claim to be "the" applied mathematics any more.
How to give a glimpse of the power of these new applications to our students? Perhaps some nonstandard mathematical activities like programming and modeling (to be discussed later) can be used here.

\section{New tools: computers and information technology}

Computers, of course, are not only sources of interesting and novel mathematical problems.

They also provide new tools for doing and organizing our research. We use them for e-mail and word processing, for experimentation, and for getting information through the web, from the MathSciNet database, Wikipedia, the Arxives, electronic journals and from home pages of fellow mathematicians.

Are these uses of computers just toys or at best matters of convenience? I think not, and that each of these is going to have a profound impact on our science.

It is easiest to see this about experimentation with Maple, Mathematica, Matlab, or your own programs. These programs open for us a range of observations and experiments which had been inaccessible before the computer age, and which provide new data and reveal new phenomena.

Electronic journals and databases, home pages of people, companies and institutions, Wikipedia, and e-mail provide new ways of dissemination of results and ideas. In a sense, they reinforce the increase in the volume of research: not only are there increasingly more people doing research, but an increasingly large fraction of this information is available at our fingertips (and often increasingly loudly and aggressively: the etiquette of e-mail is far from solid). But we can also use them as ways of coping with the information explosion.

Electronic publication is gradually transforming the way we write papers. At first sight, word processing looks like just a convenient way of writing; but slowly many features of electronic versions become available that are superior to the usual printed papers: hyperlinks, colored figures and illustrations, animations and the like.

The use of computers is an area where often we learn from our students, not the other way around. The question here is: how to use the interest and knowledge in computing, present in most students today, for the purposes of mathematical education? Most suitable for this seem to be some nonstandard mathematical activities, which I discuss next. 


\section{New forms of mathematical activity}

\section{Algorithms and programming}

The traditional 2500 year old paradigm of mathematical research is defining notions, stating theorems and proving them. Perhaps less recognized, but almost this old, is algorithm design (think of the Euclidean Algorithm or Newton's Method). While different, these two ways of doing mathematics are strongly interconnected (see [6]). It is also obvious that computers have increased the visibility and respectability of algorithm design substantially.

Algorithmic mathematics (put into focus by computers, but existent and important way before their development!) is not the antithesis of the "theorem-proof" type classical mathematics, which we call here structural. Rather, it enriches several classical branches of mathematics with new insight, new kinds of problems, and new approaches to solve these. So: not algorithmic or structural mathematics, but algorithmic and structural mathematics!

What does this imply in math education? As we discussed above, mathematical education must follow, at least to some degree, what happens in mathematical research; this is especially so in those (rare) cases when research results fundamentally change the whole framework of the subject. So set theory had to enter mathematical education (one would wish with more moderation and less controversy than happened with "new math"). Algorithmic mathematics is another one of these.

However, the range of the penetration of an algorithmic perspective in classical mathematics is not yet clear at all, and varies very much from subject to subject (as well as from lecturer to lecturer). Graph theory and optimization, for example, have been thoroughly re-worked from a computational complexity point of view; number theory and parts of algebra are studied from such an aspect, but many basic questions are unresolved; in analysis and differential equations, such an approach may or may not be a great success; set theory does not appear to have much to do with algorithms at all.

Our experience with "New Math" warns us that drastic changes may be disastrous even if the new framework is well established in research and college mathematics. Some algorithms and their analysis could be taught about the same time when theorems and their proofs first occur, perhaps around the age of 14 . Of course, certain algorithms (for multiplication and division etc.) occur quite early in the curriculum. But these are more recipes than algorithms; no correctness proofs are given (naturally), and the efficiency is not analyzed.

The beginning of learning "algorithmics" is to learn to design, rather than execute, algorithms [8]. The euclidean algorithm, for example, is one that can be "discovered" by students in class. In time, a collection of "algorithm design problems" will arise (just as there are large collec- tions of problems and exercises in algebraic identities, geometric constructions or elementary proofs in geometry). Along with these concrete algorithms, the students should get familiar with basic notions of the theory of algorithms: input-output, correctness and its proof, analysis of running time and space, etc.

In college, the shift to a more algorithmic presentation of the material should, and will, be easier and faster. Already now, some subjects like graph theory are taught in many colleges quite algorithmically: shortest spanning tree, maximum flow and maximum matching algorithms are standard topics in most graph theory courses. This is quite natural since, as I have remarked, computational complexity theory provides a unifying framework for many of the basic graph-theoretic results. In other fields this is not quite so at the moment; but some topics like primality testing or cryptographic protocols provide nice applications for a large part of classical number theory.

One should distinguish between an algorithm and its implementation as a computer program. The algorithm itself is a mathematical object; the program depends on the machine and/or on the programming language. It is of course necessary that the students see how an algorithm leads to a program that runs on a computer; but it is not necessary that every algorithm they learn about or they design be implemented. The situation is analogous to that of geometric constructions with ruler and compass: some constructions have to be carried out on paper, but for some more, it may be enough to give the mathematical solution (since the point is not to learn to draw but to provide a field of applications for a variety of geometric notions and results).

Let me insert a warning about the shortcomings of algorithmic language. There is no generally accepted form of presenting an algorithm, even in the research literature (and as far as I see, computer science text books for secondary schools are even less standardized and often even more extravagant in handling this problem.) The practice ranges from an entirely informal description to programs in specific programming languages. There are good arguments in favor of both solutions; I am leaning towards informality, since I feel that implementation details often cover up the mathematical essence. For example, an algorithm may contain a step "Select any element of set S". In an implementation, we have to specify which element to choose, so this step necessarily becomes something like "Select the first element of set S". But there may be another algorithm, where it is important the we select the first element; turning both algorithms into programs hides this important detail. Or it may turn out that there is some advantage in selecting the last element of S. Giving an informal description leaves this option open, while turning the algorithm into a program forbids it.

On the other hand, the main problem with the informal presentation of algorithms is that the "running time" or "number of steps" are difficult to define; this depends on the details of implementation, down to a level 
below the programming language; it depends on the data representation and data structures used.

The route from the mathematical idea of an algorithm to a computer program is long. It takes the careful design of the algorithm; analysis and improvements of running time and space requirements; selection of (sometimes mathematically very involved) data structures; and programming. In college, to follow this route is very instructive for the students. But even in secondary school mathematics, at least the mathematics and implementation of an algorithm should be distinguished.

An important task for mathematics educators of the near future (both in college and high school) is to develop a smooth and unified style of describing and analyzing algorithms. A style that shows the mathematical ideas behind the design; that facilitates analysis; that is concise and elegant would also be of great help in overcoming the contempt against algorithms that is still often felt both on the side of the teacher and of the student.

\section{Problems and conjectures}

In a small community, everybody knows what the main problems are. But in a community of 100,000 people, problems have to be identifed and stated in a precise way. Poorly stated problems lead to boring, irrelevant results. This elevates the formulation of conjectures to the rank of research results. Conjecturing became an art in the hands of the late Paul Erdös, who formulated more conjectures than perhaps all mathematicians before him put together. He considered his conjectures as part of his mathematical œuvre as much as his theorems.

Of course, it is diffcult to formulate what makes a good conjecture. (There is even a lot of controversy around Erdős' conjectures.) It is easy to agree that if a conjecture is good, one expects that its resolution should advance our knowledge substantially. Many mathematicians feel that this is the case when we can clearly see the place of the conjecture, and its probable solution, in the building of mathematics; but there are conjectures so surprising, so utterly inaccessible by current methods, that their resolution must bring something new-we just don't know where.

In the teaching style of mathematics which emphasizes discovery (which I personally find the best), good teachers always challenged their students to formulate conjectures leading up to a theorem or to the steps of a proof. This is time-consuming, and there is a danger that this activity too is eroding under the time pressure discussed above. I feel that it must be preserved and encouraged.

\section{Mathematical experiments}

In some respects, computers allow us to turn mathematics into an experimental subject. Ideally, mathematics is a deductive science, but in quite a few situations, experimentation is warranted:

(a) Testing an algorithm for efficiency, when the resource requirements (time, space) depend on the input in a toocomplicated way to make good predictions. ${ }^{1}$

(b) Cryptographic and other computer security issues often depend on classical questions about the distribution of primes and similar problems in number theory, and the answers to these questions often depend on notoriously difficult problems in number theory, like the Riemann Hypothesis and its extensions. Needless to say that in such practically crucial questions, experiments must be made even if deductive answers would be ideal.

(c) Experimental mathematics is a good source of conjectures; a classical example is Gauss' discovery (not proof) of the Prime Number Theorem. Among the contemporary examples of this, let me mention the most systematic one: the graph-theoretic conjecture-generating program GRAFFITI by Fajtlowicz [2, $3]$.

There are several excellent books about experimental mathematics (see e.g. [1]). Programs like Derive, Maple or Mathematica offer us, and the students, many ways of experimentation with mathematics. A simple example: a student can develop a real feeling for the notion of convergence and convergence rate by comparing the computation of the convergent sums $\Sigma 1 / k^{2}$ and $\Sigma 1 / 2^{k}$.

Mathematical experimentation has indeed been used quite extensively in the teaching of analysis, number theory, geometry, and many other topics. The success seems to be controversial; my feeling is that, similarly as in the teaching of algorithms, the development of large well-tested sets of experimental tasks takes time, and is the most crucial element of the success of these teaching methods.

\section{Modeling}

To construct good models is the most important first step in almost every successful application of mathematics. The role of modeling in education is well recognized [9], but its weight relative to other material, and the ways of teaching it, are quite controversial. Modeling is a typical interactive process, where the mathematician must work together with engineers, biologist, economists, and many other professionals seeking help from mathematics. A possible approach here is to combine teaching of mathematical modeling with education in team work and professional interaction.

A good example is the course "Discrete Mathematical Modeling" at the University of Washington [4] (similar

\footnotetext{
${ }^{1}$ I do not include here verification of the correctness of a program-which is not a mathematical issue, but a matter of software engineering.
} 
courses are taught at several other universities, e.g. at the Eötvös University in Budapest). The main feature of this course is that the students, in groups of 2 or 3, must find a real-life problem in their environment. They have to develop a model, gather data, find and code the algorithms that answer the original question, and give a presentation of the results. The real-life problems raised are quite broad in scope, from problems on favorite games to attempts to help family or friends in their business, and some of the answers obtained turn out quite useful.

\section{Exposition and popularization}

The role of this activity is growing very fast in the mathematical research community. Besides the traditional way of writing a good monograph (which is of course still highly regarded), there is more and more demand for expositions, surveys, minicourses, handbooks and encyclopedias. Many conferences (and often the most successful ones) are mostly or exclusively devoted to expository and survey-type talks; publishers much prefer volumes of survey articles to volumes of research papers. While full recognition of expository work is still lacking, the importance of it is more and more accepted.

On the other hand, mathematics education does little to prepare students for this. Mathematics is a notoriously difficult subject to talk about to outsiders (including even scientists). I feel that much more effort is needed to teach students at all levels how to give presentations, or write about mathematics they learned. (One difficulty may be that we know little about the criteria for a good mathematical survey.)

\section{References}

[1] J.M. Borwein, D.H. Bailey, R. Girgensohn:

Experimentation in Mathematics: Computational Paths to Discovery, A.K. Peters (2004).

[2] S. Fajtlowicz: On conjectures of Graffiti, Discrete Math. 72 (1988), 113-118.

[3] S. Fajtlowicz: Postscript to Fully Automated Fragments of Graph Theory,

http://math.uh.edu/ siemion/postscript.pdf

[4] Discrete Mathematical Modeling, undergraduate course at the University of Washington, http://www.math.washington.edu/ goebel/381/

[5] P. R. Halmos (1981), Applied mathematics is bad mathematics, in Mathematics Tomorrow (ed. L. A. Steen), Springer, 9-20.

[6] L. Lovász: Algorithmic mathematics: an old aspect with a new emphasis, in: Proc. 6th ICME, Budapest, J. Bolyai Math. Soc. (1988), 67-78.

[7] L. Lovász: One mathematics, The Berlin Intelligencer, Mitteilungen der Deutschen Math., Verein, Berlin (1998), $10-15$.
[8] S. Maurer (1984), Two meanings of algorithmic mathematics, Mathematics Teacher, 430-435.

[9] Modelling and Applications in Mathematics Education (eds: W. Blum, P.L. Galbraith, H.-W. Henn and M. Niss), ICMI Study No. 14, Springer, 2007.

[10] M. Niss: Quantitative Literacy and Mathematical Competencies, http://www.maa.org/Ql 